\title{
Use of Quality Indicators to Evaluate the Care of Patients with Localized Prostate Carcinoma
}

\author{
David C. Miller, M.D. ${ }^{1}$ \\ Mark S. Litwin, M.D., M.P... ${ }^{2}$ \\ Martin G. Sanda, M.D. ${ }^{1}$ \\ James E. Montie, M.D. ${ }^{1}$ \\ Rodney L. Dunn, M.s. ${ }^{1}$ \\ Jennifer Resh, m.s. ${ }^{1}$ \\ Howard Sandler, M.D. ${ }^{3}$ \\ John T. Wei, M.D. ${ }^{1,4}$
}

\begin{abstract}
${ }^{1}$ Department of Urology, University of Michigan, Ann Arbor, Michigan.

${ }^{2}$ Departments of Urology and Health Services, University of California-Los Angeles, Los Angeles, California.

${ }^{3}$ Department of Radiation Oncology, University of Michigan, Ann Arbor, Michigan.

${ }^{4}$ The Veterans Affairs Center for Practice Management and Outcomes Research, Ann Arbor Veterans Affairs Medical Center, Ann Arbor, Michigan.
\end{abstract}

Supported by United States Public Health Service grant P50CA69568 (National Cancer Institute).

James E. Montie is an investigator for Astra Zeneca and UroCor.

Address for reprints: John T. Wei, M.D., M.S., Department of Urology, Taubman Health Care Center, University of Michigan Health System, 1500 East Medical Center Drive, Ann Arbor, Ml 481090330; Fax: (734) 936-9127; E-mail: jtwei@ umich.edu

Received July 3, 2002; revision received October 24, 2002; accepted October 28, 2002.
BACKGROUND. The goal of quality assurance in health care is to preserve and improve patient care. Recently, RAND developed a set of evidence-based candidate indicators for evaluating the quality of care for patients with localized prostate carcinoma; however, the feasibility and sensitivity of these indicators have not been tested in a clinical setting. The objectives of this study were to evaluate the feasibility of measuring these quality indicators and to determine their sensitivity to change in practice patterns over time.

METHODS. One hundred sixty-eight men who presented in either 1995 or in 2000 and were treated for localized prostate carcinoma were selected randomly from the University of Michigan tumor registry. A combination of electronic data base review and explicit chart review was used to assess the feasibility of measuring compliance for each indicator. For each indicator in which assessment was feasible, compliance with the RAND indicators was determined for patients in both years. Multivariate regression analysis was used to adjust for potential confounding effects of disease stage, tumor grade, prostate specific antigen (PSA) level, patient age, and therapy.

RESULTS. Based on review of available clinical data, measurement of compliance was feasible for 19 of 22 RAND candidate quality indicators (86\%). For five indicators, significant differences in documentation (compliance) were detected between 1995 and $2000(P<0.05)$. Treatment received and higher PSA levels were associated independently with documentation of compliance for several indicators $(P<0.05)$.

CONCLUSIONS. Measurement of the majority of the RAND quality indicators for the treatment of patients with localized prostate carcinoma was feasible, and improvements in several indicators were observed between 1995 and 2000. Demonstration of such variation, even within a single institution, suggests that the indicators are sufficiently sensitive to detect differences in practice patterns. Cancer 2003;97: 1428-35. (C) 2003 American Cancer Society.

DOI 10.1002/cncr.11216

KEYWORDS: prostate carcinoma, quality indicators, quality of care, quality assurance.

n 2002, it is projected that 189,000 men will have been diagnosed with prostate carcinoma, and the majority of these men will have localized disease. ${ }^{1}$ Given the large number of patients who select active treatment, ${ }^{2}$ the need for proper evaluation of the quality of care for patients with localized prostate carcinoma is particularly germane. Urinary, sexual, and bowel dysfunction are known complications of both radical prostatectomy and external beam radiation therapy. However, the reported complication rates for each treatment modality vary significantly among individual surgeons and institutions. ${ }^{3-6}$ Although this variation may be explained, at least partially, by con- 
founding patient and disease characteristics (casemix), these disparate outcomes may also reflect differences in the quality of care.

The foundation for much of the emerging research in quality assessment is based on Avedis Donabedian structure-process-outcome paradigm. ${ }^{7}$ The Donabedian paradigm is based on the ability of each component of this model to provide insight regarding the degree to which the best available treatment plans were used in an optimal fashion. ${ }^{7,8}$ The structure domain evaluates the infrastructure and resources of the care provider; the process domain examines what actually is done for patients and how this compares with what should be done; and the outcome domain considers the results attained by patients after medical care. $^{8,9}$

Historically, quality assessment initiatives have been driven primarily by economic forces. ${ }^{9}$ However, physicians have recognized the importance of critically analyzing patient outcomes and identifying factors that contribute to improvements in health care delivery. ${ }^{10,11}$ Indeed, clinicians have played instrumental roles in the development and implementation of benchmarks for clinical care as well as efforts to bring local practices up to national or best-practice standards. ${ }^{9,12}$

Recently, a multidisciplinary RAND team developed a set of candidate quality indicators for evaluating the quality of care for men with localized prostate carcinoma. Those results are summarized in a report entitled Prostate cancer patient outcomes and choice of providers: development of an infrastructure for quality assurance $^{13}$ and available free on line (http://www. rand.org/publications/MR/MR1227/). Based on literature review, patient focus groups, expert interviews, and a RAND consensus panel, the project established a measurement framework with a group of 22 indicators proposed for use in quality assessment. The RAND indicators provide a basis for assessing quality care for patients with localized prostate carcinoma, although the indicators have not been validated to date in a clinical setting. Therefore, the objectives of this study were 1) to evaluate the feasibility of measuring compliance with the 22 RAND candidate quality indicators in a hospital setting; and 2) for indicators for which assessment was feasible, to determine the sensitivity to changes in patterns of care.

\section{MATERIALS AND METHODS}

Under Institutional Review Board (IRB) approval, a cross-sectional review of care for patients with localized prostate carcinoma was performed for two separate calendar years (1995 and 2000), representing early and contemporary care for patients with prostate car- cinoma at the University of Michigan. One hundred patients with localized prostate carcinoma who presented to our institution in each of these 2 years were selected randomly from the hospital tumor registry for chart abstraction. Thirty-two of 200 randomly selected patients were excluded from the study. Exclusion criteria included incidental prostate adenocarcinomas discovered at the time of cystoprostatectomy performed for urothelial carcinoma, primary therapy other than radical prostatectomy or external radiation therapy, treatment at another institution, and missing patient medical records. Based on these criteria, 19 patients, including 8 patients whose paper medical records could not be located (and electronic medical records were not yet instituted), were excluded from the 1995 cohort, and 13 patients were excluded from the 2000 cohort.

The RAND quality indicators include 4 measures of structure (S1-S4), 12 measures of process (P1-P12), and 6 measures of outcome $(\mathrm{O} 1-\mathrm{O} 6)$ (Table 1). ${ }^{13} \mathrm{~A}$ combination of electronic and paper chart review, along with data extraction from an IRB-approved prostate cancer data base, was utilized for our first objective of assessing the feasibility of measuring the 22 candidate quality indicators. These data sources were reviewed for documentation of each quality indicator (Table 1). For the purpose of this study, measurement of a particular quality indicator was considered feasible only if explicit documentation was identified for at least one patient. For each indicator for which measurement of compliance was feasible, the proportion of patients with documentation (of compliance) was then reported and stratified by year. A chart abstraction tool was created in Microsoft Access software to record all relevant data. All chart reviews were performed by two independent investigators (D.C.M. and J.R.) who were not involved with the care of the patients.

Feasibility assessment for several of the candidate indicators (S1-S3) was based on institutional standards and resources (Table 1). In addition, compliance with indicator S4 was measurable for any given patient by review of our prostate cancer data base. For each structure measure, the corresponding resources and/or services have been available for all patients at the University of Michigan since at least 1995. Therefore, measurement of compliance with each structure indicator was feasible for all patients who were included in our analysis.

Feasibility assessment for several process measures (P8, P13, and P14) required examination of paper and/or electronic medical records (Table 1). Process measure P8 assessed whether or not the patient was presented with the various treatment options for 
TABLE 1

RAND Quality Indicators for the Care of Patients with Localized Prostate Carcinoma: Sources of Documentation for Feasibility Assessment

\begin{tabular}{|c|c|c|c|c|c|}
\hline Indicator & Description & Institutional $^{\mathrm{a}}$ & $\begin{array}{l}\text { Paper } \\
\text { medical } \\
\text { record }^{b}\end{array}$ & $\begin{array}{l}\text { Electronic } \\
\text { medical } \\
\text { record }^{\mathrm{c}}\end{array}$ & $\begin{array}{l}\text { Prostate } \\
\text { cancer } \\
\text { data base }^{\mathrm{d}}\end{array}$ \\
\hline \multicolumn{6}{|c|}{ Structure indicators } \\
\hline S1 & No. of patients treated (volume) & $\mathrm{X}^{\mathrm{e}}$ & - & - & - \\
\hline S2 & Availability of conformal RT & $\mathrm{X}^{\mathrm{e}}$ & - & - & - \\
\hline S3 & Availability of psychological counseling & $\mathrm{X}^{\mathrm{e}}$ & - & - & - \\
\hline S4 & Knowledge of treating institution outcomes & - & - & - & $\mathrm{X}^{\mathrm{e}}$ \\
\hline \multicolumn{6}{|c|}{ Process indicators } \\
\hline P5 & Pretreatment PSA, DRE, Gleason score & - & $\mathrm{X}$ & $\mathrm{X}$ & $\mathrm{X}^{\mathrm{e}}$ \\
\hline $\mathrm{P} 6$ & $\begin{array}{l}\text { Documentation of pretreatment urinary, sexual, and bowel } \\
\text { function }\end{array}$ & - & - & - & $\mathrm{X}^{\mathrm{e}}$ \\
\hline P7 & Assessment of family history of prostate carcinoma & - & $\mathrm{X}$ & $\mathrm{X}$ & $\mathrm{X}^{\mathrm{e}}$ \\
\hline P8 & $\begin{array}{l}\text { Presentation of treatment alternatives; consultation with alternative } \\
\text { providers; discussion of risks of therapy }\end{array}$ & - & $\mathrm{X}^{\mathrm{e}}$ & $X^{e}$ & - \\
\hline P9 & $\begin{array}{l}\text { Adherence to practice protocol of the College of American } \\
\text { Pathologists Cancer Committee }\end{array}$ & - & - & - & $\mathrm{X}^{\mathrm{e}}$ \\
\hline P10 & $\begin{array}{l}\text { Use of immobilization, CT planning and optimal dosing (68-72 Gy) } \\
\text { for patients undergoing conventional external RT }\end{array}$ & $\mathrm{NF}^{\mathrm{f}}$ & $\mathrm{NF}^{\mathrm{f}}$ & $\mathrm{NF}^{\mathrm{f}}$ & $\mathrm{NF}^{\mathrm{f}}$ \\
\hline P11 & $\begin{array}{l}\text { Use of immobilization, CT planning, rectal protection and optimal } \\
\text { dosing (70-80 Gy) for patients undergoing high-dose conformal } \\
\text { RT }\end{array}$ & $\mathrm{NF}^{\mathrm{f}}$ & $\mathrm{NF}^{\mathrm{f}}$ & $\mathrm{NF}^{\mathrm{f}}$ & $\mathrm{NF}^{\mathrm{f}}$ \\
\hline P12 & For RT: Use of high-energy linear accelerator ( $\geq 10 \mathrm{MV}$ ) & $\mathrm{NF}^{\mathrm{f}}$ & $\mathrm{NF}^{\mathrm{f}}$ & $\mathrm{NF}^{\mathrm{f}}$ & $\mathrm{NF}^{\mathrm{f}}$ \\
\hline $\mathrm{P} 13$ & $\begin{array}{l}\text { At least two follow-up visits with the treating physician in the first } \\
\text { year posttherapy }\end{array}$ & - & $\mathrm{X}^{\mathrm{e}}$ & $\mathrm{X}^{\mathrm{e}}$ & - \\
\hline P14 & $\begin{array}{l}\text { Documentation of communication with primary care provider or } \\
\text { provision of continuing care }\end{array}$ & - & $\mathrm{X}^{\mathrm{e}}$ & $\mathrm{X}^{\mathrm{e}}$ & - \\
\hline P15 & Operative blood loss & - & $\mathrm{X}$ & $\mathrm{X}$ & $\mathrm{X}^{\mathrm{e}}$ \\
\hline $\mathrm{P} 16$ & Use of clinical and pathologic TNM staging & - & $\mathrm{X}$ & $\mathrm{X}$ & $\mathrm{X}^{\mathrm{e}}$ \\
\hline \multicolumn{6}{|c|}{ Outcome indicators } \\
\hline 017 & $\begin{array}{l}\text { Primary treatment failure indicated by three consecutive increases } \\
\text { in PSA after RT or any detectable PSA after RP }\end{array}$ & - & - & - & $\mathrm{X}^{\mathrm{e}}$ \\
\hline 018 & $\begin{array}{l}\text { After RT: Hospitalization, medical or surgical, treatment for cystitis, } \\
\text { proctitis, hematuria, or rectal bleeding }\end{array}$ & - & - & - & $\mathrm{X}^{\mathrm{e}}$ \\
\hline 019 & $\begin{array}{l}\text { After RT or RP: Hospitalization, medical or surgical treatment for } \\
\text { bladder neck contracture/urethral stricture }\end{array}$ & - & - & - & $\mathrm{X}^{\mathrm{e}}$ \\
\hline 020 & Acute surgical complication rate & - & - & - & $\mathrm{X}^{\mathrm{e}}$ \\
\hline 021 & $\begin{array}{l}\text { Patient assessment of urinary, sexual, and bowel function after RT } \\
\text { or RP using a validated survey instrument }\end{array}$ & - & - & - & $\mathrm{X}^{\mathrm{e}}$ \\
\hline 022 & Patient satisfaction with treatment choice, continence, and potency & - & - & - & $\mathrm{X}^{\mathrm{e}}$ \\
\hline \\
\hline & & & \multicolumn{3}{|c|}{ a Institutional refers to the availability of resources maintained by the institution. } \\
\hline \multicolumn{6}{|c|}{ b Paper medical record refers to the official hard-copy medical record maintained by the Medical Records Department. } \\
\hline \multicolumn{6}{|c|}{ ' Electronic medical record refers to the official computer-based medical record maintained by the Medical Records Department. } \\
\hline \multicolumn{6}{|c|}{$\begin{array}{l}\text { d Prostate cancer data base refers to the research data base maintained by the Department of Urology for clinical research and quality assurance. } \\
\text { e Primary source of documentation }\end{array}$} \\
\hline \multicolumn{6}{|c|}{ e Primary source of documentation } \\
\hline${ }^{\mathrm{f}}$ Measuremen & pliance was not feasible based on a review of the available medical records data or & & & & \\
\hline
\end{tabular}

localized prostate carcinoma as well as the risks of therapy. Measurement of this indicator was feasible, in that compliance can be inferred from a single sentence in the clinician's note such as, "treatment options and their associated risk were discussed in detail with the patient." Similarly, feasibility assessment for measures P13 and P14 also required explicit review of electronic and paper medical records, because this information was not available in our data base. For this measure, letters of communication from treating physicians to primary care providers were deemed sufficient to achieve compliance, confirming the measurability of P14.

In contrast, the measurement of one process indicator (P9) and several outcome indicators (O17, O18, O19, and O20) was feasible only by review of our prostate cancer data base (Table 1), because the documentation necessary to confirm or refute compliance 
with each of these measures was contained therein. P9, for instance, evaluates an institution's adherence to the prostate carcinoma practice protocol of the College of American Pathologists (CAP) Cancer Committee. ${ }^{14}$ For the purposes of this study, compliance with this indicator could be measured by review of the abstracted pathology report for each patient in our prostate cancer data base. Notably, there are several minor elements of this protocol that are not documented routinely for patients at our institution (urethral obstruction in prostatectomy specimens and extracapsular extension for pathologic lymph nodes). Inclusion of these elements was not considered necessary for compliance, because they comprise only a limited proportion of the entire CAP protocol; furthermore, these findings were documented when present. Outcome measures O17, O18, O19, and O20 evaluate biochemical recurrence and complication rates after therapy. The feasibility of measuring these indicators was determined by review of our electronic data base. A data management specialist reviewed each clinic note for patients who were treated with radiation therapy or radical prostatectomy, and relevant information, such as recent prostate specific antigen (PSA) levels and therapy-related complications, were identified and entered accordingly. Therefore, measurement of compliance with $\mathrm{O} 17, \mathrm{O} 18, \mathrm{O} 19$, and $\mathrm{O} 20$ was feasible, because the rigorous chart review process utilized to maintain our data base captures the occurrence of significant clinical events, including biochemical recurrences, therapy-related complications, and/or hospitalizations.

Three of the candidate indicators (P6, O21, and O22) assessed quality-of-life issues and patient satisfaction. The validated instrument currently in use at the University of Michigan for patient assessment of urinary, sexual, and bowel function after primary therapy is the Expanded Prostate Cancer Index Composite (EPIC) ${ }^{15}$ EPIC results are maintained routinely in our prostate cancer data base. Therefore, measurement of compliance with candidate indicators $\mathrm{P} 6, \mathrm{O} 21$, and $\mathrm{O} 22$ was feasible by querying the prostate cancer data base (Table 1).

Feasibility assessment for several other RAND indicators (P5, P7, P10, P11, P12, P15, and P16) was accomplished by a combination of electronic data base review and explicit chart review (Table 1). Measurement of compliance with process indicators P5, P7, P15, and P16 was feasible primarily by review of our prostate cancer data base. P5 assessed pretherapy documentation of serum PSA level, digital rectal examination (DRE) findings, and Gleason sum. These data were verified for nearly all patients by electronic query of our prostate cancer data base. In patients for whom one of these staging parameters was not available in our data base, explicit review of the patient's electronic and paper records yielded the necessary documentation (confirmation of compliance). Documentation of family history assessment (P7), operative blood loss (P15), and use of clinical and pathologic TNM staging (P16) also were determined primarily from electronic data base review. Once again, in patients for whom a field was blank in our data base, explicit chart review was undertaken to confirm or refute compliance with each of these process measures.

Candidate indicators $\mathrm{P} 10, \mathrm{P} 11$, and $\mathrm{P} 12$ reviewed several aspects of the quality of radiation therapy provided for patients with localized prostate carcinoma. Evaluation of the indicator P10 was not possible at our institution, because no patients who were included in our analysis received conventional external radiation therapy.

The second objective of this study was to compare differences in compliance between 1995 and 2000 for those indicators for which assessment was feasible. For each indicator for which a change in the documentation (compliance) was observed, bivariate comparison of the proportions between the 2 years was performed using the Fisher exact test. Multivariable logistic regression analysis was then used to adjust for potential confounding effects due to differences in patient age (years), clinical stage (T1 vs. T2 or higher), biopsy grade (Gleason sum), pretreatment PSA level (ng/mL), and treatment received (radical prostatectomy vs. external beam radiation therapy). ${ }^{16}$ All hypothesis testing was done at the $5 \%$ significance level, and all statistical analyses were performed using SAS software (version 8.0; SAS Institute, Inc., Cary, NC).

\section{RESULTS}

Table 2 summarizes the demographics, clinical stage, and treatment for the final cohort of 168 patients who were included in the analyses. These characteristics did not differ by year (all $P$ values $>0.05$ ).

\section{Feasibility of Measuring Compliance with the RAND Candidate Indicators}

First, the feasibility of measuring compliance with the 22 RAND candidate quality indicator variables was assessed. Measurement of compliance was feasible for several of the RAND structural indicators (S1-S3), because they simply reflected available institutional resources and services (Table 1). For example, the second structure measure (S2) evaluates the availability of conformal radiation therapy at the treating institution. At our institution, conformal radiation is provided as the standard of care for patients undergoing 
TABLE 2

Characteristics of the Study Population Stratified by the Year Treatment was Provided

\begin{tabular}{lll}
\hline Characteristic & $\begin{array}{l}\mathbf{1 9 9 5} \\
(\boldsymbol{n}=\mathbf{8 1} \text { patients })\end{array}$ & $\begin{array}{l}\mathbf{2 0 0 0} \\
(\boldsymbol{n}=\mathbf{8 7} \text { patients })\end{array}$ \\
\hline Mean \pm SD age (yrs) & $63.2 \pm 7.3$ & $60.5 \pm 9.5$ \\
Mean \pm SD pretreatment PSA (ng/dL) & $8.1 \pm 9.1$ & $8.8 \pm 10.0$ \\
Caucasian (\%) & 90.1 & 83.9 \\
Clinical stage (\%) & & \\
T1 & 57.0 & 76.0 \\
T2 & 42.0 & 23.0 \\
T3 & 1.0 & 1.0 \\
Prostatectomy (\%) & 77.8 & 78.2 \\
\hline
\end{tabular}

a SD: standard deviation; PSA: prostate specific angiten.

radiation therapy since 1986 . Therefore, this indicator is considered measurable in all patients, because it is available to all patients who pursue radiation therapy.

Measurement of compliance with the majority of the indicators (S4, P5-P9, P13-P16, and O17-O22) was feasible using a combination of electronic data base and/or explicit review of the official medical records (paper and electronic) (Table 1). Assessment of pretreatment and posttreatment urinary, sexual, and bowel function (P6 and O21) was feasible only by review of our prostate cancer data base. In general, although thorough, explicit chart review yielded documentation of most of the indicators, our study was facilitated greatly by the availability of a prostate cancer data base.

Measurement of compliance was not feasible for three of the RAND process indicators (P10, P11, and P12). Feasibility assessment for process indicator P10 was not possible because no patients in this study received conventional radiation therapy. In addition, measurement of compliance with process measures P11 and P12 was not feasible, because the clinical data (i.e., use of immobilization, computed tomographic planning, and rectal protection) required to confirm or refute compliance with these indicators is not maintained routinely in either the official medical record or our prostate cancer data base.

\section{Sensitivity of the RAND Indicators to Detect Changes in Practice Patterns}

Several of the RAND candidate indicators (S1-S4, P9, $\mathrm{P} 15, \mathrm{O} 17-\mathrm{O} 20$, and O22) had identical levels of compliance (either $0 \%$ or $100 \%$ ) both in 1995 and in 2000 . In contrast, there were several process and outcome measures (P6-P8, P13, P14, P16, and O21) for which changes in compliance were detected between 1995 and 2000. For those indicators for which differences

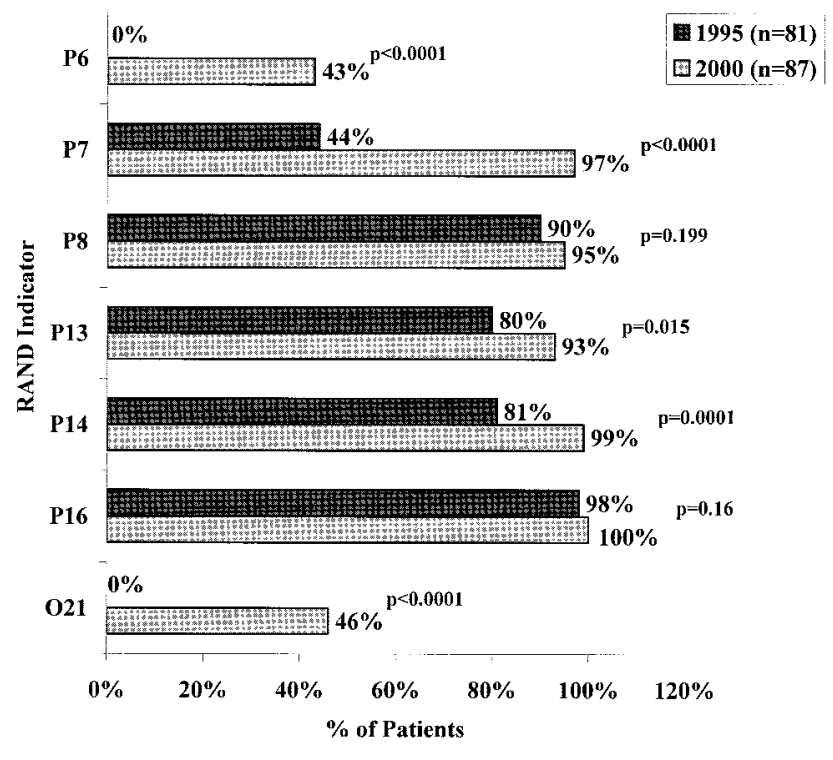

FIGURE 1. Compliance with 7 of 22 RAND indicators varied between 1995 and 2000. Compliance with structure indicators S1-S4; with process indicators $\mathrm{P} 5, \mathrm{P} 9, \mathrm{P} 10, \mathrm{P} 11, \mathrm{P} 12$, and P15; and with outcome indicators 017, 018, 019, and 020 was $100 \%$ in both years; whereas compliance with outcome indicator 022 was $0 \%$ in both years.

were observed, the proportion of patients with documentation (of compliance) in each year and the corresponding statistical significance are presented in Figure 1. Notably, although no patients had comprehensive documentation of pretreatment urinary, sexual, and bowel function (P6) in 1995, many patients did have documentation for one or two of these domains. For the purposes of our analyses, this was considered insufficient documentation to achieve compliance with indicator P6.

Multivariable logistic regression models were constructed for each indicator for which changes in practice patterns were noted. For several indicators (P6, P7, P13, and O21), we found that higher PSA level and type of therapy received were associated independently with compliance (Tables 3, 4). Patient age, clinical stage, and biopsy grade did not affect compliance with these candidate indicators.

\section{DISCUSSION}

Patients and advocacy groups are increasingly interested in the documentation of medical care quality. ${ }^{10}$ In addition, the government and major medical societies have invested tremendous effort in quality assurance, as evidenced by the rigorous standards established and enforced by routine Joint Commission on Accreditation of Healthcare Organizations audits. The universal goal of all such initiatives is to establish standards that optimize the quality of patient care. 
TABLE 3

Quality Indicator for which Compliance Was Associated Significantly with Higher Pretreatment Prostate Specific Antigen Levels ${ }^{\mathrm{a}}$

\begin{tabular}{llllll}
\hline & & & \multicolumn{3}{c}{ Quartiles of pretreatment PSA levels (ng/mL) } \\
\cline { 3 - 6 } Indicator & RAND quality indicator & Q1 (0.1-3.4) & Q2 (3.5-5.7) & Q3 (5.8-9.7) & Q4 (9.8-73.7) \\
\hline P6 & Documentation of pretreatment urinary, sexual, and bowel function & $27 \%$ & $30 \%$ & $24 \%$ & $7 \%$ \\
\hline \begin{tabular}{l} 
a Percentages represent the proportion of patients in compliance with this indicator. \\
\hline
\end{tabular}
\end{tabular}

TABLE 4

Quality Indicators for which Compliance Was Associated Significantly with Treatment Received $(P<0.05)^{\mathrm{a}}$

\begin{tabular}{llll}
\hline & & & Treatment received (\%) \\
\cline { 3 - 4 } Indicator & RAND quality indicator & Prostatectomy & External radiation \\
\hline P6 & Documentation of pretreatment urinary, sexual and bowel function & 28 & 3 \\
P7 & Documentation of family history & 64 & 97 \\
P13 & At least two follow-up visits after therapy & 90 & 74 \\
P21 & Patient assessment of urinary, sexual, and bowel function after RT & 31 & 0 \\
& or RP using a validated survey instrument & & \\
\hline
\end{tabular}

Recently, RAND developed 22 candidate indicators that provide an objective framework for measuring and monitoring quality of care for patients undergoing therapy for localized prostate carcinoma. The indicators are evidence based and are consistent with the principles of the recent National Cancer Policy Board report concerning the assessment of quality care for oncology patients. ${ }^{13}$ The RAND report recommended performing additional studies before the RAND criteria can be considered the infrastructure for quality care assessments in patients with prostate carcinoma. In the current study, we evaluated the feasibility of measuring compliance with the 22 RAND candidate quality indicators in a hospital setting and determined their sensitivity to changes in the care of patients with localized prostate carcinoma.

The availability of electronic data sources played a vital role in documenting compliance with many of the RAND candidate indicators. We took advantage of the existing prostate cancer data base at the University of Michigan to collect data for many of the RAND process measures. Specific information abstracted from the data base included documentation of pretreatment PSA level, Gleason sum, clinical stage, and form of therapy. These data have been used previously to identify determinants of better health outcomes after patients are treated for prostate carcinoma. ${ }^{17-19}$ Our results support the concept that development and maintenance of comprehensive, longitudinal data bases can substantially augment and simplify the doc- umentation of quality indicators. In sum, through a process of explicit review of both medical records and existing data bases, we demonstrated that, for patients with localized prostate carcinoma, measurement of compliance was feasible for 19 of 22 RAND candidate indicators. The feasibility of measuring compliance was unambiguous for most of the indicators and supports their application in future quality assessment studies.

Between 1995 and 2000, based on the documentation in available clinical data, changes in compliance were detected for several of the process indicators. During this interval, we demonstrated significantly improved compliance with assessment of family history of prostate carcinoma (P7), frequency of posttherapy follow-up visits (P13), and communication with primary care physicians $(\mathrm{P} 14)(P<0.05$ for each). Moreover, we identified documentation of pretreatment urinary, sexual, and bowel function (P6) as a deficiency in our process of care. Indeed, compliance with documentation of pretreatment function was lacking for all patients who were treated in 1995; however, $43 \%$ of patients were in compliance with this indicator in 2000. This improvement coincides with our current practice, beginning in July 2000, of routinely administering the EPIC questionnaire prior to radical prostatectomy.

In both 1995 and 2000, 100\% compliance with documentation was achieved for four of six outcome indicators (O17-O20). Documentation of these events 
(biochemical failures and complications of surgical and/or radiation therapy) is maintained rigorously in readily accessible data bases, as noted above. It may be relevant to point out that the RAND report does not specify an optimal follow-up period for tracking therapeutic failures and complications. In this context, clarification of an optimal follow-up interval will enhance the validity of these indicators and will facilitate the assessment of compliance with these outcome measures in future investigations.

However, we also identified several weaknesses in our institutional approach to outcomes assessment, as reflected in our level of compliance with indicators O21 and O22. In 1995, no validated survey instruments were in use at our center for patient assessment of urinary, sexual, and bowel function after treatment with radiation therapy or radical prostatectomy $(\mathrm{O} 21)$. Thus, although its measurement is feasible, compliance with this indicator was absent uniformly in 1995. During the intercurrent period, our interest in measuring outcomes led to the development of novel, validated instruments for this purpose (EPIC). ${ }^{15}$ Accordingly, compliance with documentation of posttreatment function increased from $0 \%$ to $46 \%$ between 1995 and $2000(P<0.0001)$. Now, health-related quality-of-life data are obtained routinely for all patients treated at our institution to further enhance our understanding of functional outcomes after surgery. Consequently, we anticipate that the nearly all patients who undergo radical prostatectomy at the University of Michigan in subsequent years will have documentation, using a validated instrument, of both preoperative and postoperative urinary, sexual, and bowel function. Efforts to achieve similar compliance among patients who receive radiation therapy currently are underway but have not yet achieved uniform implementation.

Another area for potential quality improvement relates to assessment of patient satisfaction (O22). Although measurement of this indicator was feasible, the observed lack of compliance with indicator $\mathrm{O} 22$ reflects the absence of validated tools for evaluating posttreatment patient satisfaction with their overall care as well as their oncologic and functional outcomes. Thus, efforts to systematically measure patient satisfaction as an indicator of quality care must include the development of a validated instrument for this purpose.

In this study, we assumed that practice patterns at our institution changed between 1995 and 2000. Indeed, during this period, assessment of outcomes prompted substantial changes in the process of care for patients with localized prostate carcinoma. ${ }^{18-21}$ In this context, the demonstrated variation in several of the candidate indicators reflects these changes in practice patterns and supports the concept that the RAND indicators are potentially sensitive to differences in quality of care. Moreover, the ability to detect such variation within a single institution suggests that the proposed quality indicators may be useful tools for large-scale, interinstitutional comparisons of quality care.

However, other factors also may affect quality of care and should be considered in conjunction with these indicators. In our study, we observed that higher pretreatment PSA levels were associated with whether or not pretreatment urinary, sexual, and bowel function were documented, indicating that patients with higher PSA levels were less compliant with our prospective quality-of-life evaluative studies. Treatment received also was associated with compliance for several of the candidate indicators, suggesting that physician bias or specialty bias may affect documentation or performance of these quality indicators. Taken together, these finding substantiate the importance of controlling for various disease-related, physician-related, and therapy-related factors when evaluating quality of care.

Several limitations to this study merit discussion. First, the RAND report did not propose standards for documentation of compliance with each indicator. Therefore, it was necessary for us to establish institution specific standards. Without clear guidelines for documenting compliance, the reliability for certain indicators may be limited in future multiinstitutional studies. In addition, the absence of such guidelines may limit the ability of nonphysician or nonmedical personnel to abstract this information from the chart, a transition that will be critical to the widespread implementation of assessments of quality care for patients with prostate carcinoma. Indeed, future studies should compare, on a larger scale, the findings of physician and nonphysician abstracters to ensure the validity and reliability of the data.

A second limitation to consider relates to pathologic evaluation of prostatectomy specimens. In this study, we documented compliance with the CAP Prostate Cancer protocol for all patients. Two minor elements from this protocol were not documented routinely but, instead, were reported only when present. This suggests a need to modify the CAP protocol as a quality indicator to make it more practical (P6).

Our inability to measure compliance with candidate indicators P10, P11, and P12 also merits further deliberation. Each of these indicators evaluates the quality of radiation therapy provided for patients with localized prostate carcinoma. Despite a thorough review of electronic and paper medical records, as well 
as our prostate cancer data base, measurement of compliance was not feasible for these indicators. Thus, although compliance with chemotherapy planning, immobilization, rectal protection (P11), and the use of a high-energy accelerator (P12) may have been achieved based on institutional protocols, we were unable to confirm or refute this for any patient, despite a rigorous review of available clinical data. This suggests that, when they are performed, routine documentation of these radiotherapeutic details will be necessary before they are established as indicators of quality.

A final limitation relates to insufficient detail in assessing pretreatment patient function. Although compliance with documentation of pretreatment urinary, sexual, and bowel function (P6) was lacking uniformly in 1995, the vast majority of patients had documentation for one or two of these domains. However, this distinction cannot be made with indicator P6 in its current form, because compliance with this indicator requires documentation of urinary, sexual, and bowel function. Therefore, P6 may underestimate pretherapy assessments, because many patients who, in fact, were queried regarding pretreatment functional status are reported as not in compliance due to lack of documentation for all three domains. Once again, this suggests that our ability to evaluate quality care, as well as to detect different practice patterns among urologists and radiation oncologists, may require refinement of certain quality indicators.

\section{CONCLUSIONS}

We have demonstrated the feasibility of measuring compliance with the RAND candidate quality indicators in a clinical hospital setting and have demonstrated the ability of these indicators to detect changes in quality of care over time. Future efforts to refine the RAND indicators, develop benchmarks and to assess quality of care on a larger scale have the potential to lead to improved health outcomes for patients with localized prostate carcinoma.

\section{REFERENCES}

1. Jemal A, Thomas A, Murray T, et al. Cancer statistics, 2002. CA Cancer J Clin. 2002;52:23-47.

2. Demers RY, Tiwari A, Wei JT, et al. Trends in the utilization of androgen-deprivation therapy for patients with prostate carcinoma suggest an effect on mortality. Cancer. 2001;92: 2309-2317.

3. Litwin MS, Hays RD, Fink PA, et al. Quality-of-life outcomes in men treated for localized prostate cancer. JAMA. 1995; 273:129-135.

4. Talcott JA, Rieker P, Clark JA, et al. Patient-reported symptoms after primary therapy for early prostate cancer: results of a prospective cohort study. J Clin Oncol. 1998;16:275-283.

5. Middleton RG, Thompson IM, Austenfeld WH, et al. Prostate Cancer Clinical Guidelines Panel summary report on the management of clinically localized prostate cancer. The American Urological Association. J Urol. 1995;154:21442148.

6. Brandeis JM, Litwin MS, Burnison CM, et al. Quality of life outcomes after brachytherapy for early stage prostate cancer. J Urol. 2000;163:851-857.

7. Donabedian A. The role of outcomes in quality assessment and assurance. Qual Rev Bull. 1992;18:356-360.

8. Donabedian A. The seven pillars of quality. Arch Pathol Lab Med. 1990;114:1115-1118.

9. Christianson SW. Gaining the competitive edge: why quality counts. Semin Med Pract. 1998;1:11-15.

10. Bernstein AB. Ready or not, here it comes: medical practice in the new millennium. Semin Med Pract. 1998;1:2-6.

11. Blumenthal D. Quality of health care part 1. Quality of care: what is it? N Engl J Med. 1996;335:891-894.

12. Ullman M, Metzger CK, Kuzel T, et al. Performance measurement in prostate cancer care: beyond report cards. Urology. 1996;47:356-365.

13. Litwin MS, Steinberg M, Malin J, et al. Prostate cancer patient outcomes and choice of providers: development of an infrastructure for quality assessment. Santa Monica: RAND, 2000.

14. Srigley JR, Amin MB, Bostwick DG, et al. Updated protocol for the examination of specimens from patients with carcinomas of the prostate gland: a basis for checklists. Cancer Committee. Arch Pathol Lab Med. 2000;124:1034-1039.

15. Wei JT, Dunn RL, Litwin MS, et al. Development and validation of the Expanded Prostate Cancer Index Composite (EPIC) for comprehensive assessment of health-related quality of life in men with prostate cancer. Urology. 2000; 56:899-905.

16. Agresti A. Categorical data analysis. New York: John Wiley \& Sons Inc., 1990.

17. Montie JE. Quality assurance in urologic surgery: the radical prostatectomy experience. Ann Arbor: Department of Surgery Grand Rounds, University of Michigan, 2000.

18. Kielb SJ, Dunn RL, Rashid MG, et al. Assessment of early continence recovery after radical prostatectomy: patient reported symptoms and impairment. J Urol. 2001;166:958961.

19. Wei JT, Dunn RL, Marcovich R, et al. Prospective assessment of patient reported urinary continence after radical prostatectomy. J Urol. 2000;164:744-748.

20. Goh M, Kleer C, Kielczewski P, et al. Autologous blood donation prior to anatomical radical retropubic prostatectomy: is it necessary? Urology. 1997;49:569-573.

21. Marcovich R, Wojno KJ, Oesterling JE, et al. Bladder-neck sparing modification of radical prostatectomy adversely effects surgical margins in pathologic T3a prostate cancer. Urology. 2000;55:904-908. 